\title{
Frequency comparison of light transmission in a defected quasi-one-dimensional photonic crystal slab
}

\author{
Rostam Moradian ${ }^{1,2,3}$ and Jamileh Samadi ${ }^{1,2^{*}}$
}

\begin{abstract}
In a new theoretical investigation, we study light transmission through a photonic crystal (PC) slab with limited boundaries at width. By using a tight binding model, photon dispersion relation and photon Green function for a perfect system are obtained. Then, based on the Lippmann-Schwinger formalism, we calculate the effects of disordering on light transmission in the PC channel. We found that the ratio of the electric field for a defected system with respect to a perfect system at a peculiar frequency is maximized for the wave vector corresponding to the first Brillouin zone (BZ) edge showing photon localization. The electric field difference of the first and second neighboring sites with respect to the defect site on the first BZ edges are depicted in several plots indicating frequency dependence which can be applicable in frequency filtering or resonating cavity studies.
\end{abstract}

Keywords: Photonic crystals, Lippmann-Schwinger equation, Photon green function, Disordered structures

\section{Background}

The design of two-dimensional (2D) or three-dimensional periodic dielectric arrangements with proper choice of materials, lattice symmetry, embedded defect creation, and boundary situations in photonic crystals (PCs) enables the control of light flow at an elevated level. PC research is one of the most interesting and applicable issues. Since the pioneer proposals for inhibition of light emission [1] and localization of light [2], different approaches on PCs have been investigated. In recent experimental research, more attention has been given to photon localization in PC microcavities and wave guides [3-5], nanocavities [6], and disordered or amorphous PCs [7-9]. Theoretical investigations have mostly used perturbation theory completed with Green tensor [10,11], tight binding method [12-14], Wannier function [15], and Lippmann-Schwinger formalism [16]. Well-engineered designs and proper materials lead to less refraction at the

\footnotetext{
*Correspondence: jamilehsamadi@yahoo.com

1 Department of Physics, Faculty of Science, Razi University, Kermanshah

67149-67346, Iran

2 Nano Science and Technology Research Center, Razi University, Kermanshah 67149-67346, Iran

Full list of author information is available at the end of the article
}

boundaries; therefore, light transports through the bulk by interference in the internal structure while the occurrence of boundaries affects the dispersion relation as reported for metallic PC resonators [17] and also limited boundaries with antireflection structures [18]. Here our task is the investigation of light transmission along the length of a perfected PC slab with a width limited by a highdielectric-constant material at boundaries. Vanishing of the electric field of incident light at the boundaries due to total reflection leads to quantization of photon modes for the perpendicular wave vector. Then, by exhibiting a defect on one of the PC slab sites, we show that light transmission completely differs for special modes at the defect and its neighboring sites. The ratio of incident modes of defected systems with respect to clean systems can be calculated from the difference of these individual modes by applying the appropriate photonic Green function. The organization of the paper is as follows: In the 'Methods' section, we extended the method implemented in [12] to discretize Maxwell's equations of our system. One of these equations is used for transverse magnetic (TM) modes to introduce an eigenvalue-eigenfunction equation of the PC slab in tight binding method. Then, photonic Green function and photonic dispersion relation are obtained for the

\section{它 Springer}

(C) 2013 Moradian and Samadi; licensee Springer. This is an Open Access article distributed under the terms of the Creative Commons Attribution License (http://creativecommons.org/licenses/by/2.0), which permits unrestricted use, distribution, and reproduction in any medium, provided the original work is properly cited. 
described system. In the last step, defected system electric field is related to the perfect system electric field by applying an equation based on the Lippmann-Schwinger formalism. In the'Results and discussion' section, application of this equation is investigated by several plots of the electric field and intensity at the defect site and its neighboring sites for different cases, followed by the the 'Conclusions' section.

\section{Methods}

\section{Discretization of Maxwell's equations}

The structure under our consideration is a PC system consisting of periodic dielectric rods with finite thickness in a two-dimensional slab. Figure 1a shows a PC slab sandwiched between two high-dielectric-constant layers parallel to the $x$-axis direction. Also, due to total reflection, the electric field vanishes at the boundaries. Although the slab is limited in width, the $x$-axis direction is unlimited. A point defect can be easily exhibited by inserting an air rod instead of one of the dielectric lattice sites (Figure 1b) while the nearest neighboring dielectric rods are numbered. We begin our investigation by following Maxwell's equations for electric field $E$ and magnetic field $H$ :

$$
\begin{aligned}
& \frac{1}{\varepsilon(r)} \nabla \times\{\nabla \times E(r)\}=\frac{\omega^{2}}{c^{2}} E(r), \\
& \nabla \times\left\{\frac{1}{\varepsilon(r)} \nabla \times H(r)\right\}=\frac{\omega^{2}}{c^{2}} H(r),
\end{aligned}
$$

where $\omega$ is the light frequency, $c$ is the light velocity, and $\varepsilon_{r}(r)$ is the dielectric constant. By assuming that the dielectric constant $\varepsilon_{r}(r)$ is independent of the $z$ coordinate, we rewrite the first equation for a two-dimensional slab. We just study the TM mode since the mentioned PC structure with dielectric rods is appropriate for TM modes $[19,20]$. This formalism is general, and for TE modes the same procedures have to be reviewed. Extending the method implemented in [12], we discretize Equation 1 since for TM modes the main components of fields are $\left(E_{z}, H_{x}, H_{y}\right)$; therefore, the electric field and the other two
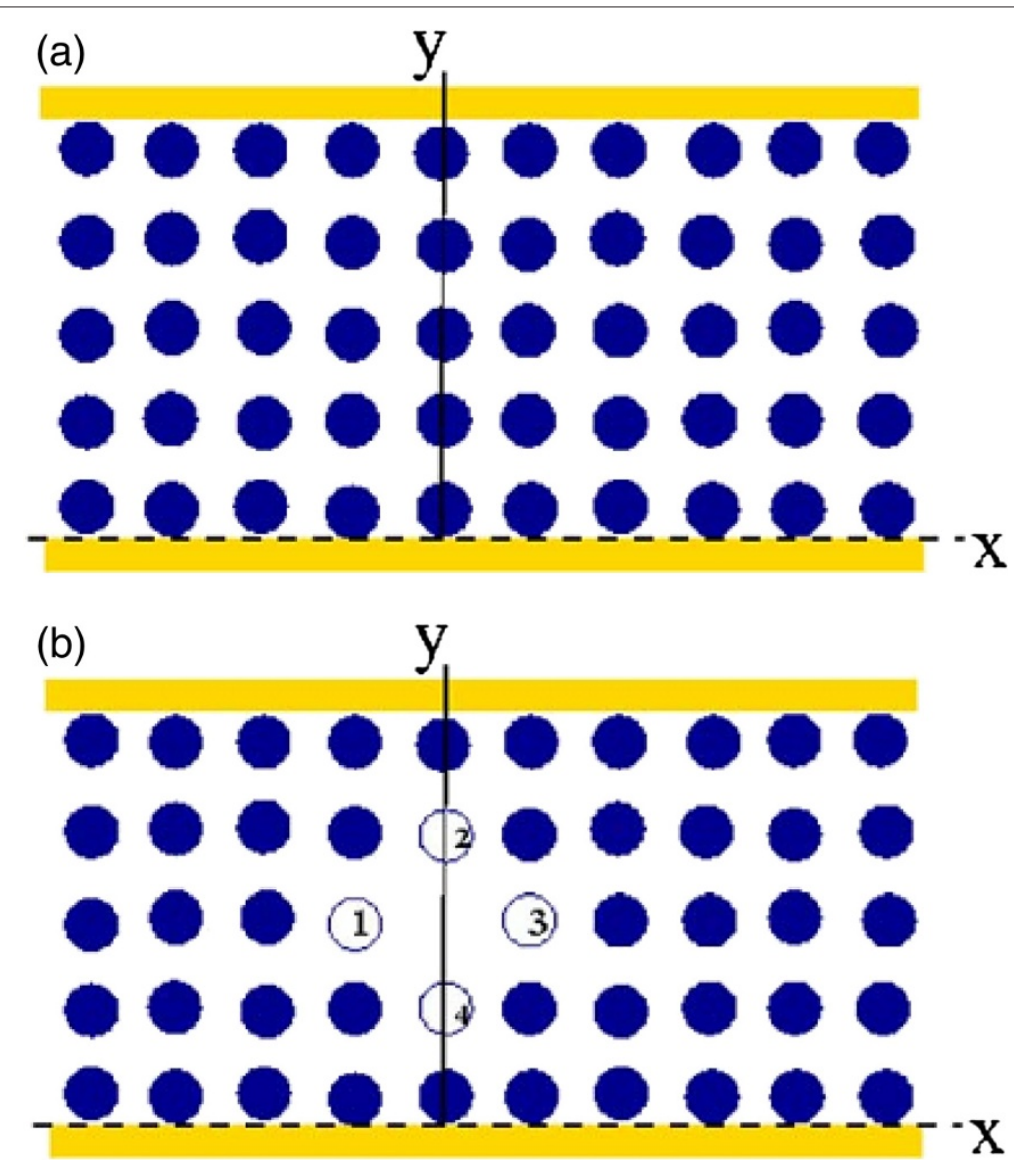

Figure 1 A PC slab. A PC slab consisting of high-dielectric rods between low-dielectric background which is sandwiched in two high-dielectric-constant layers parallel to the $x$-axis direction, (a) the perfect system and (b) the imperfect system, while the central rod is removed. The neighboring sites are not removed but are indicated by numbers. 
components can be solved by the following differential equations:

$$
\begin{aligned}
& \frac{1}{\varepsilon(r)}\left(\frac{\partial^{2} E_{z}}{\partial x^{2}}+\frac{\partial^{2} E_{z}}{\partial y^{2}}\right)+\frac{\omega^{2}}{c^{2}} E_{z}=0, \\
& H_{x}=\frac{-i}{\omega \mu_{0}} \frac{\partial E_{z}}{\partial y}, \quad H_{y}=\frac{i}{\omega \mu_{0}} \frac{\partial E_{z}}{\partial x} .
\end{aligned}
$$

The corresponding equation for $E_{z}$ in the form of operator equation with a Hermitian differential operator could be defined as [12]

$$
L_{\mathrm{TM}} f=\left(\frac{\omega}{c}\right)^{2} f
$$

where $L$ and the function $f$ for TM modes are defined by

$$
\begin{aligned}
L_{\mathrm{TM}} & =-\frac{1}{\sqrt{ } \varepsilon(r)}\left(\frac{\partial^{2} E_{z}}{\partial x^{2}}+\frac{\partial^{2} E_{z}}{\partial y^{2}}\right) \frac{1}{\sqrt{ } \varepsilon(r)}, \\
f & =\sqrt{ } \varepsilon_{r}(r) E_{z} .
\end{aligned}
$$

This equation has to be discretized in the $x$ direction, and the position of the center of the $l$ th site in the $x$ direction is indicated by $x_{l}=l a_{0}$ and in the $y$ direction by $y^{\alpha}=\alpha a_{0}$, where $a_{0}$ is the lattice constant and $l$ and $\alpha$ are integers. While $l$ is an infinite number, $\alpha$ is finite. By using the following discretization of Equation 5,

$$
\begin{aligned}
& a_{0}^{2} \frac{\partial^{2}}{\partial x^{2}} \xi(x) f(x) \quad \rightarrow \quad \xi_{l+1}^{\alpha} f_{l+1}^{\alpha}-2 \xi_{l}^{\alpha} f_{l}^{\alpha}+\xi_{l-1}^{\alpha} f_{l-1}^{\alpha}, \\
& a_{0}^{2} \frac{\partial^{2}}{\partial y^{2}} \xi(y) f(y) \quad \rightarrow \quad \xi_{l}^{\alpha+1} f_{l}^{\alpha+1}-2 \xi_{l}^{\alpha} f_{l}^{\alpha}+\xi_{l}^{\alpha-1} f_{l}^{\alpha-1},
\end{aligned}
$$

where $f_{l}^{\alpha}=\sqrt{ } \xi_{r, l}^{\alpha} E_{z, l}^{\alpha}$ and $\xi_{l}^{\alpha}=\frac{1}{\sqrt{ } \xi_{r, l}^{\alpha}}$, then a finite differential equation is obtained:

$$
\begin{array}{r}
v_{l}^{\alpha} f_{l}^{\alpha}-t_{l, l+1}^{\alpha, \alpha} f_{l+1}^{\alpha}-t_{l, l-1}^{\alpha, \alpha} f_{l-1}^{\alpha}-t_{l, l}^{\alpha, \alpha+1} f_{l}^{\alpha+1}-t_{l, l}^{\alpha, \alpha-1} f_{l}^{\alpha-1} \\
=\left(\frac{\omega a_{0}}{c}\right)^{2} f_{l}^{\alpha},
\end{array}
$$

where the coefficients are defined as

$$
\begin{aligned}
& v_{l}^{\alpha}=4\left(\xi_{l}^{\alpha}\right)^{2}, \quad t_{l, l+1}^{\alpha, \alpha}=\xi_{l}^{\alpha} \xi_{l+1}^{\alpha}, \quad t_{l, l-1}^{\alpha, \alpha}=\xi_{l-1}^{\alpha} \xi_{l}^{\alpha}, \\
& t_{l, l}^{\alpha, \alpha+1}=\xi_{l}^{\alpha+1} \xi_{l}^{\alpha}, \quad t_{l, l}^{\alpha, \alpha-1}=\xi_{l}^{\alpha} \xi_{l}^{\alpha-1} .
\end{aligned}
$$

\section{General form of tight binding model for a PC slab}

For mapping of light propagation in a PC to a tight binding model, we introduce photon creation and annihilation operators $a_{l}^{\alpha \dagger}$ and $a_{l}^{\alpha}$ for the site $\left(l a_{0}, \alpha a_{0}\right)$ with the following bosonic particle commutation relations:

$$
\left[a_{l}^{\alpha}, a_{l}^{\alpha^{\prime} \dagger}\right]=\delta_{l, l^{\prime}}^{\alpha, \alpha^{\prime} \dagger}, \quad\left[a_{l}^{\alpha \dagger}, a_{l}^{\alpha^{\prime} \dagger}\right]=0, \quad\left[a_{l}^{\alpha}, a_{l^{\prime}}^{\alpha^{\prime}}\right]=0 .
$$

When the Hermitian operator $\widehat{L}$ acts on the photonic field stateket $\mid f>$, we have the following eigenvalueeigenfunction equation:

$$
\widehat{L}\left|f>=\left(\frac{\omega a_{0}}{c}\right)^{2}\right| f>,
$$

where $\widehat{L}$ and $\mid f>$ are defined by

$$
\begin{aligned}
& \widehat{L}=\sum_{l ; \alpha}\left(v_{l}^{\alpha} a_{l}^{\alpha \dagger} a_{l}^{\alpha}\right)-\sum_{l, m ; \alpha, \beta}\left(t_{l, m}^{\alpha, \beta} a_{l}^{\alpha \dagger} a_{l}^{\alpha}\right), \\
& \left|f>=\sum_{l ; \alpha} f_{l}^{\alpha} a_{l}^{\alpha \dagger}\right| 0>,
\end{aligned}
$$

where $\mid 0>$ is the photonic vacuum state. The result is equivalent to the finite differential equation (7). To distinguish defected and perfect systems, index zero is used for the perfect system. Equation 11, in terms of perfect system operators and onsite and hopping energies, is given by

$$
\widehat{L}=\widehat{L}_{0}+\sum_{l ; \alpha}\left(v_{l}^{\prime \alpha}-v_{l}^{\alpha}\right) a_{l}^{\alpha \dagger} a_{l}^{\alpha}-\sum_{l, m ; \alpha, \beta}\left(t_{l, m}^{\prime \alpha, \beta}-t_{l, m}^{\alpha, \beta}\right) a_{l}^{\alpha \dagger} a_{m}^{\beta},
$$

where prime $v_{l}^{\prime \alpha}=4\left(\xi_{l}^{\prime \alpha}\right)^{2}$ and $t_{l, m}^{\alpha, \beta}=\xi_{l}^{\alpha} \xi_{m}^{\prime \beta}$. For convenience, two parentheses on the right-hand side of Equation 13 are substituted with $\varepsilon_{l}^{\alpha}$ and $\delta t_{l, m}^{\alpha, \beta}$, respectively:

$$
\widehat{L}=\widehat{L}_{0}+\sum_{l ; \alpha} \varepsilon_{l}^{\alpha} a_{l}^{\alpha \dagger} a_{l}^{\alpha}-\sum_{l, m ; \alpha, \beta} \delta t_{l, m}^{\alpha, \beta} a_{l}^{\alpha \dagger} a_{m}^{\beta} .
$$

The matrix element of Equation 14 is $L_{l, m}^{\alpha, \beta}=L_{0 l, m}^{\alpha, \beta}+$ $\varepsilon_{l}^{\alpha} \delta_{l, m}^{\alpha, \beta}-\delta t_{l, m}^{\alpha, \beta}$. This equation could be written in the following operator form:

$$
\widehat{L}=\widehat{L}_{0}+\widehat{v},
$$

where $\widehat{v}$ is defined by

$$
\widehat{v}=\widehat{\varepsilon}-\widehat{\delta t} .
$$

Now an efficient propagator, the Green function [21], is defined:

$$
\begin{aligned}
& \left(\left(\frac{\omega a_{0}}{c}\right)^{2}-\widehat{L}\right) G(\omega)=\widehat{1} \\
& G^{0}(\omega)=\left(\left(\frac{\omega a_{0}}{c}\right)^{2}-\widehat{L}_{0}\right)^{-1} .
\end{aligned}
$$

In the following part, we determine the Green functions for the described system to calculate the defected slab electric field.

\section{PC slab Green function}

According to Equation 10, we act the operator $\widehat{L}_{0}$ on stateket $\mid f_{k}^{0}>$, and since the eigenvalue equals $\left(\frac{\omega_{k} a_{0}}{c}\right)^{2}$, 


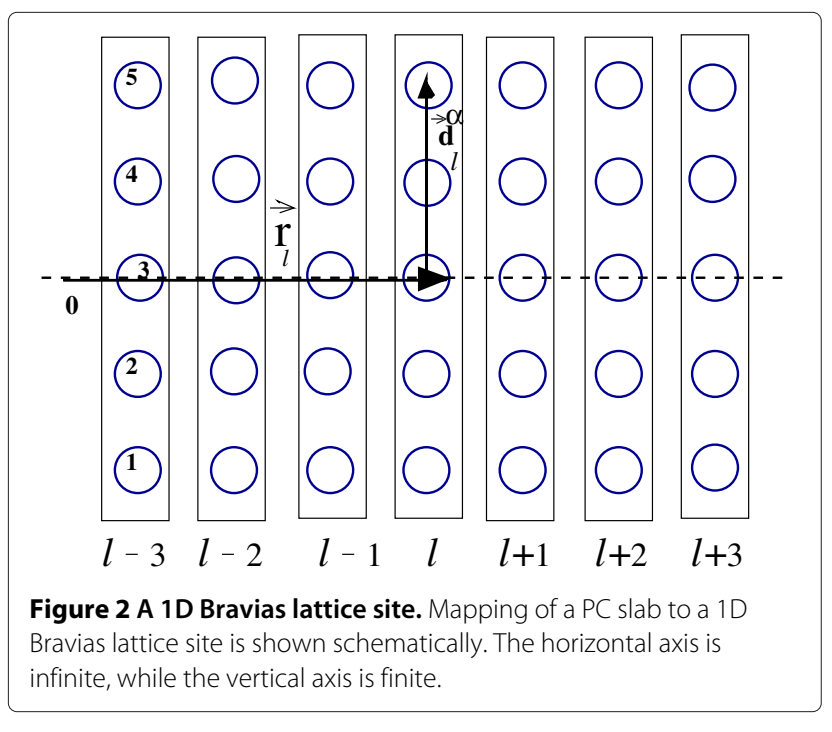

we proceed a quantum mechanical treatment to obtain the PC slab Green function:

$$
\left(E-\widehat{L}_{0}\right)\left|f_{k}^{0}>=\left(\left(\frac{\omega a_{0}}{c}\right)^{2}-\left(\frac{\omega_{k} a_{0}}{c}\right)^{2}\right)\right| f_{k}^{0}>,
$$

where $E=\left(\frac{\omega a_{0}}{c}\right)^{2}$ and $k$ characterizes the field mode. Including all modes, we have

$$
\begin{gathered}
\left(E-\widehat{L}_{0}\right) \sum_{k}\left(\frac{1}{\left(\frac{\omega a_{0}}{c}\right)^{2}-\left(\frac{\omega_{k} a_{0}}{c}\right)^{2}}\right)\left|f_{k}^{0}><f_{k}^{0}\right| \\
=\sum_{k}\left|f_{k}^{0}><f_{k}^{0}\right|,
\end{gathered}
$$

then the Green function is defined:

$$
G^{0}(k)=\sum_{k}\left(\frac{1}{\left(\frac{\omega a_{0}}{c}\right)^{2}-\left(\frac{\omega_{k} a_{0}}{c}\right)^{2}}\right)\left|f_{k}^{0}><f_{k}^{0}\right| .
$$
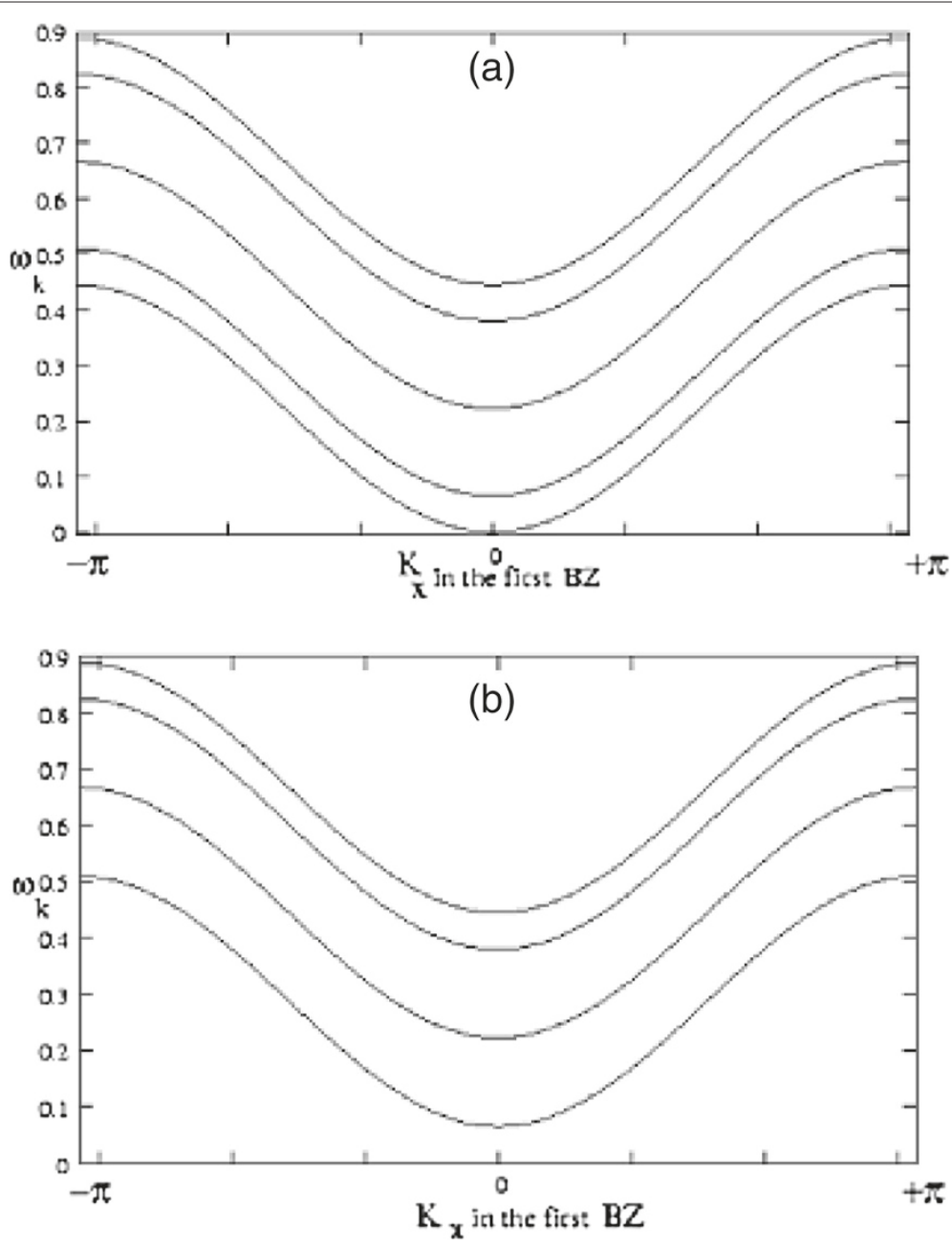

Figure 3 The dispersion relations for different integer numbers. Dispersion relation in terms of $k_{x}$ is plotted for two different numerical integers of $v$ corresponding to $k_{y}=\frac{\pi v}{w}$. Mode numbers start upward to higher values. (a) $v=0,1,2,3,4$. There is no degeneracy for modes. (b) $v=1,2,3,4,5$. The third and fifth modes are degenerate. 


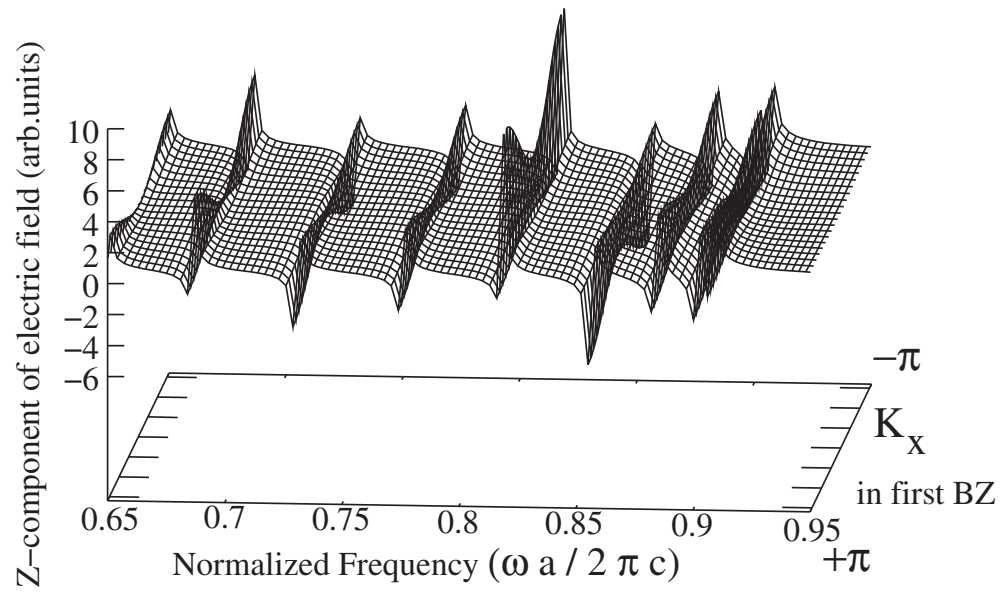

Figure 4 The ratio of electric field for defect site. The ratio of electric field with respect to its clean system at defect site $/=0$ in sub-site $\alpha=3$ (central site) is plotted. The peak's height is maximum 10.2 at frequency 0.819 corresponding to the edges of the first BZ (the third axis).

The field stateket $\mid f>$ or the electric field ket can be determined according to [20], but since the electric field vanishes at the limited boundaries, the following relation is adequate for the electric field stateket:

$$
\begin{aligned}
\mid f_{k_{x}, v}\left(x_{l}^{\alpha}, y_{l}^{\alpha}\right)>= & \sum_{l, v} C_{k_{x}, v} \exp \left(i k_{x} x_{l}^{\alpha}\right) \\
& \times \sin \left(\frac{\pi v}{w} y_{l}^{\alpha}\right) \mid u_{k_{x}, v}\left(x_{l}^{\alpha}\right)>,
\end{aligned}
$$

where $C_{k_{x}, v}$ is the normalization factor, $v$ is an integer indicating discrete modes along $y$ axes, and $w$ is the slab width. By defining $\vec{r}$ as a one-dimensional (1D) Bravias lattice site position and $\vec{d}_{l}^{\alpha}$ as a sub-site position with respect to the 1D Bravias lattice site in such a description as Figure 2 which shows mapping of a PC slab to a 1D Bravis lattice site, the electric field ket Equation 19 is also written as

$$
\begin{aligned}
\mid f_{k_{x}, \nu}(\vec{r})>= & \sum_{l, v, \alpha} C_{k_{x}, \nu} \exp \left(i k_{x} x_{l}^{\alpha}\right) \\
& \times \sin \left(\frac{\pi v}{w} \vec{d}_{l}^{\alpha}\right) \mid u_{k_{x}, \nu}\left(\vec{r}-\vec{r}_{l}-\vec{d}_{l}^{\alpha}\right)>;
\end{aligned}
$$

therefore, a general relation for the perfect slab Green function component is

$$
\begin{aligned}
G_{l, m}^{0 \alpha, \beta}= & \sum_{k_{x}, v}\left(\frac{1}{\left(\frac{\omega a_{0}}{c}\right)^{2}-\left(\frac{\omega_{k} a_{0}}{c}\right)^{2}}\right)\left|C_{k_{x}, v}\right|^{2} \\
& \times<u_{k_{x}, v}\left(\vec{r}-\vec{r}_{l}-\vec{d}_{l}^{\alpha}\right) \mid f_{k_{x}, v}\left(x_{l}^{\gamma}, y_{l}^{\gamma}\right)> \\
& \times<f_{k_{x}, v}\left(x_{m}^{\theta}, y_{m}^{\theta}\right) \mid u_{k_{x}, v}\left(\vec{r}-\vec{r}_{l}-\vec{d}_{l}^{\beta}\right)>;
\end{aligned}
$$

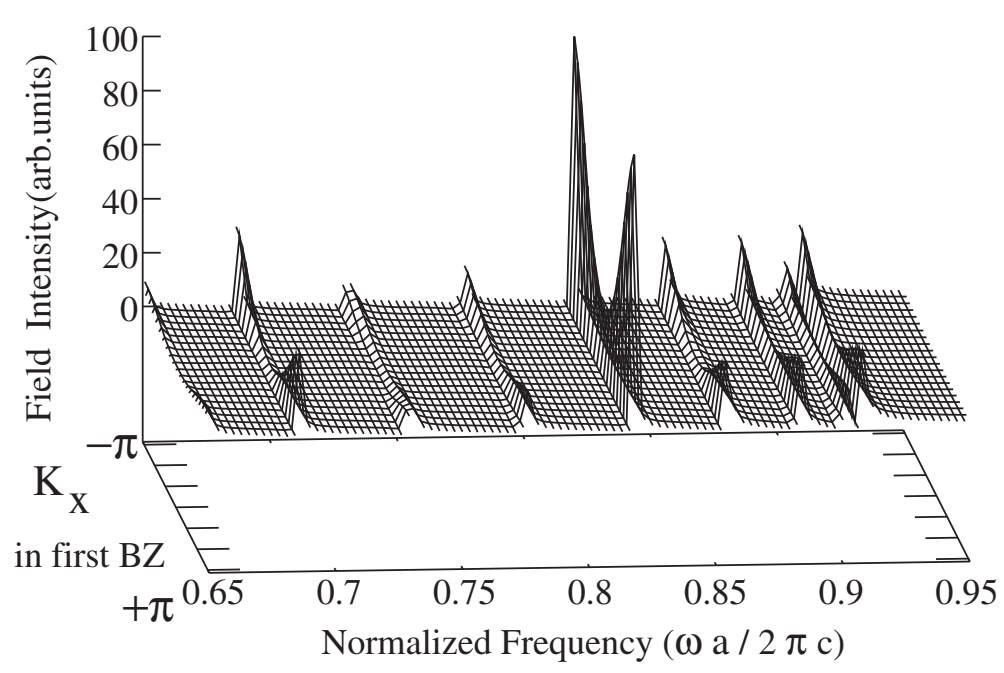

Figure $\mathbf{5}$ The ratio of field intensity at defect site. The ratio of electric field intensity with respect to its clean system at defect site is plotted. The peak's height is maximum 103 at frequency 0.819 . 


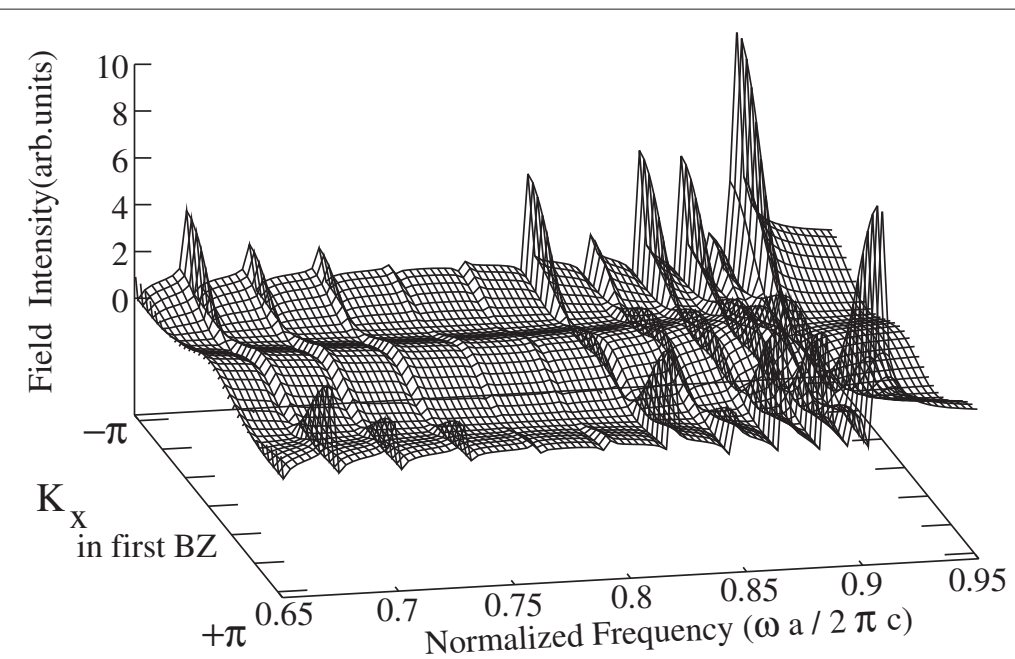

Figure 6 The ratio of field intensity for the first neighboring site of right/left side of defect site. The ratio of field intensity with respect to its clean system for the site $I= \pm 1$ and sub-site $\alpha=3$ (right side or left side of the defect site) is presented. The peak's height is maximum 10.0, with $\frac{E_{z}}{E_{z}^{\circ}}=-3.2$ at normalized frequency 0.909 .

however, for the case of the electric ket Equation 19, we have a simpler form of this component:

$$
\begin{aligned}
G_{l, m}^{0 \alpha, \beta}= & \sum_{k_{x}, v}\left(\frac{1}{\left(\frac{\omega a_{0}}{c}\right)^{2}-\left(\frac{\omega_{k} a_{0}}{c}\right)^{2}}\right)\left|C_{k_{x}, v}\right|^{2} \exp \left[i k_{x}\left(x_{l m}^{\alpha \beta}\right)\right] \\
& \times \sin \left(\frac{\pi v}{w} y_{l}^{\alpha}\right) \sin \left(\frac{\pi v}{w} y_{m}^{\beta}\right) .
\end{aligned}
$$

This Green function, is used to relate that defected system field to the perfect system field by the LippmannSchwinger formalism.

\section{Lippmann-Schwinger equation}

The electric field component in the $z$-axis direction, $\psi\left(\vec{r}_{l}^{\alpha}\right)$, is determined by multiplying $<u_{k_{x}, v}\left(\vec{r}-\vec{r}_{l}-\vec{d}_{l}^{\alpha}\right) \mid$ from the left to the stateket $\mid f_{k_{x}, v}\left(x_{l}^{\alpha}, d_{l}^{\alpha}\right)>$ :

$$
\psi\left(\vec{r}_{l}^{\alpha}\right)=<u_{k_{x}, v}\left(\vec{r}-\vec{r}_{l}-\vec{d}_{l}^{\alpha}\right) \mid f_{k_{x}, v}\left(x_{l}^{\alpha}, d_{l}^{\alpha}\right)>\text {; }
$$

inserting Equation 19, we have

$$
\psi\left(\vec{r}_{l}^{\alpha}\right)=C_{k_{x}, v} \exp \left(i k_{x} x_{l}^{\alpha}\right) \sin \left(\frac{\pi v}{w} d_{l}^{\alpha}\right)
$$

Perturbation modifies the electric field, but with first-order approximation of the Lippmann-Schwinger

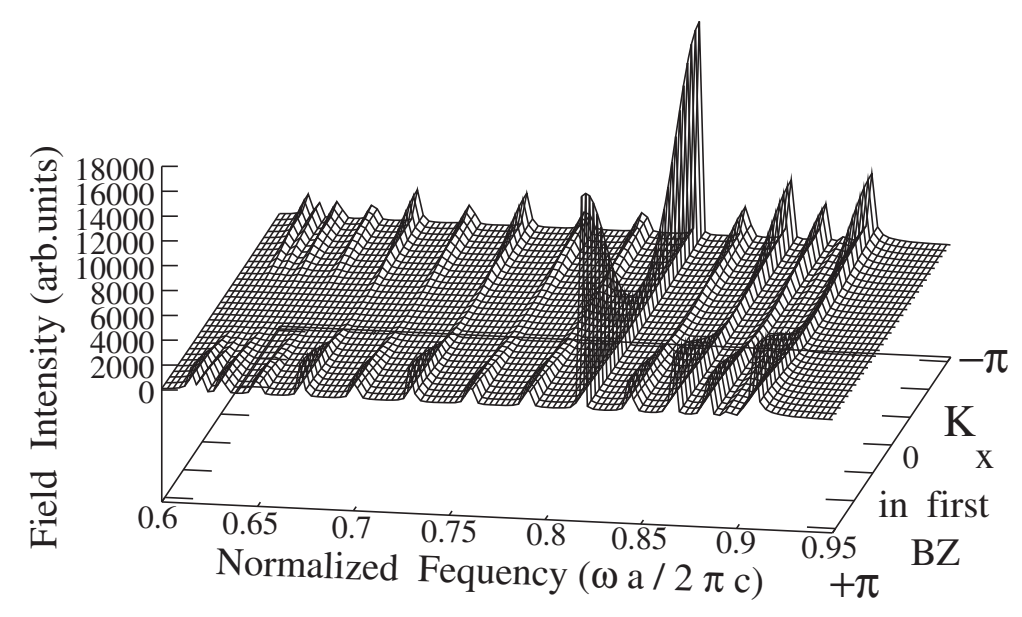

Figure 7 The field intensity for defect site. The ratio of electric field intensity with respect to its clean system at defect site, corresponding to $v=1,2,3,4,5$ of Figure $3 \mathrm{~b}$. The peak's height is maximum 17,100 , with $\frac{E_{z}}{E_{z}^{0}}=130.8$ at frequency 0.819 . 


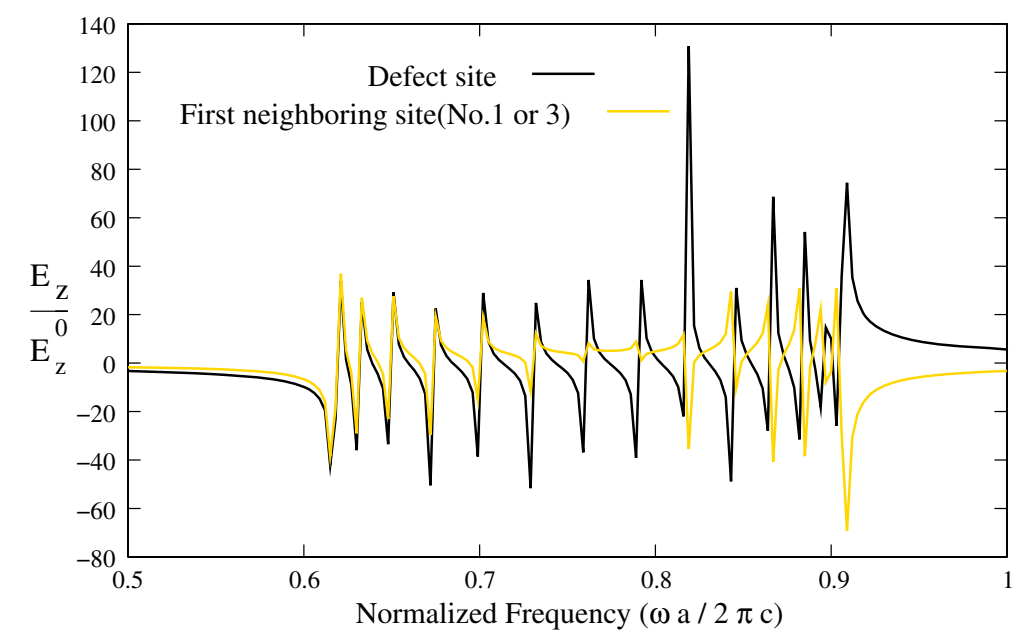

Figure 8 A comparison of electric fields for different sites. The ratio of electric field of defect site (black line) and the first neighboring site on right or left side of the defect (yellow line) are compared which indicates similarity at lower frequencies but difference at higher frequencies. $k_{x}$ is constant corresponding to the first BZ edge.

formalism, we relate the defected system electric field to perfect system's. For this purpose, we write

$$
\left(G^{0^{-1}}-v\right) \psi_{l}=\left(\left(\frac{\omega a_{0}}{c}\right)^{2}-\left(\frac{\omega_{k} a_{0}}{c}\right)^{2}\right) \psi_{l} .
$$

Using $\psi_{l} \cong \psi_{l}^{0}$ and $\omega_{k} \cong \omega_{k}^{0}$, we have $L_{0} \psi_{l} \cong\left(\frac{\omega_{k} a_{0}}{c}\right)^{2} \psi_{l}$ and

$$
\left(\left(\frac{\omega a_{0}}{c}\right)^{2}-\left(\frac{\omega_{k} a_{0}}{c}\right)^{2}\right)=\left(\left(\frac{\omega a_{0}}{c}\right)^{2}-L_{0}\right)=G^{0^{-1}},
$$

where the right-hand side of Equation 25 is equal to $G^{0-1} \psi_{l}$; then, all ends to the Lippmann-Schwinger equation:

$$
\widehat{\psi}_{l}=\widehat{\psi}_{l}^{0}+G^{0} v \widehat{\psi}_{l}^{0} .
$$

For calculating $G^{0}$, we should have the proper dispersion relation of the slab that is illustrated in Figure 1. Therefore, by Equations 7 and 19 and some mathematics, we obtain the desired relation:

$$
\begin{aligned}
\omega_{k_{x}, v}=\frac{c}{a_{0}}\left(v_{l}^{\alpha}-\right. & 2 t_{l, l \pm 1}^{\alpha, \alpha} \cos \left(k_{x} a_{0}\right) \\
& \left.-2 t_{l, l}^{\alpha, \alpha \pm 1} \cos \left(\frac{\pi v}{w} a_{0}\right)\right)^{\frac{1}{2}},
\end{aligned}
$$

which is based on tight binding method and is applicable only for the described slab.

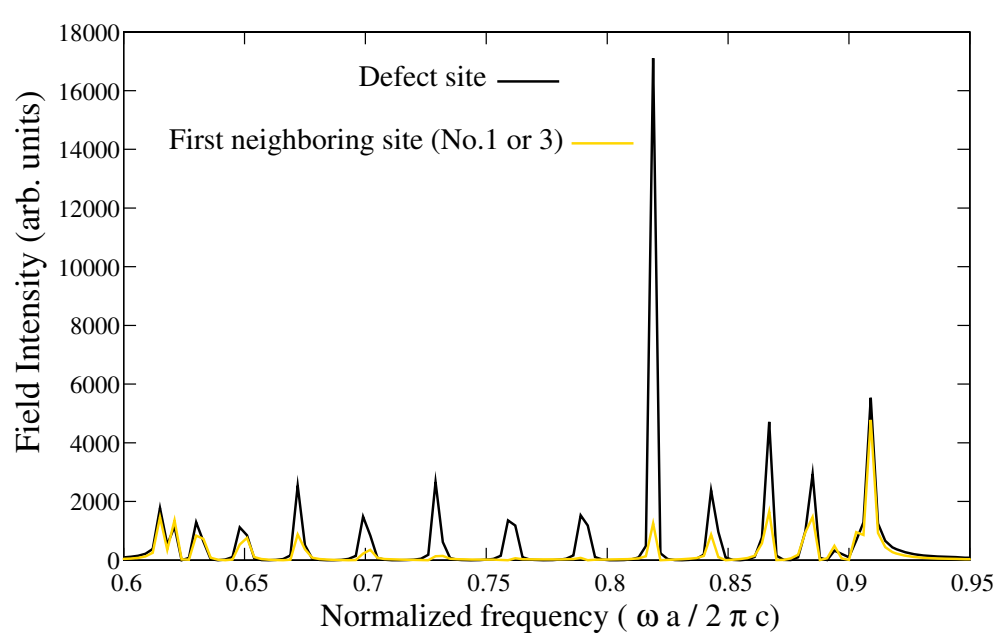

Figure 9 A comparison of the ratio of field intensity for different sites. The ratio of electric field intensity of defect site (black line) and the first neighboring site on the right or left side of the defect (yellow line) are compared which indicates that the maximum field intensity of the neighboring site is at higher frequency. $k_{x}$ is constant corresponding to the first BZ edge. 


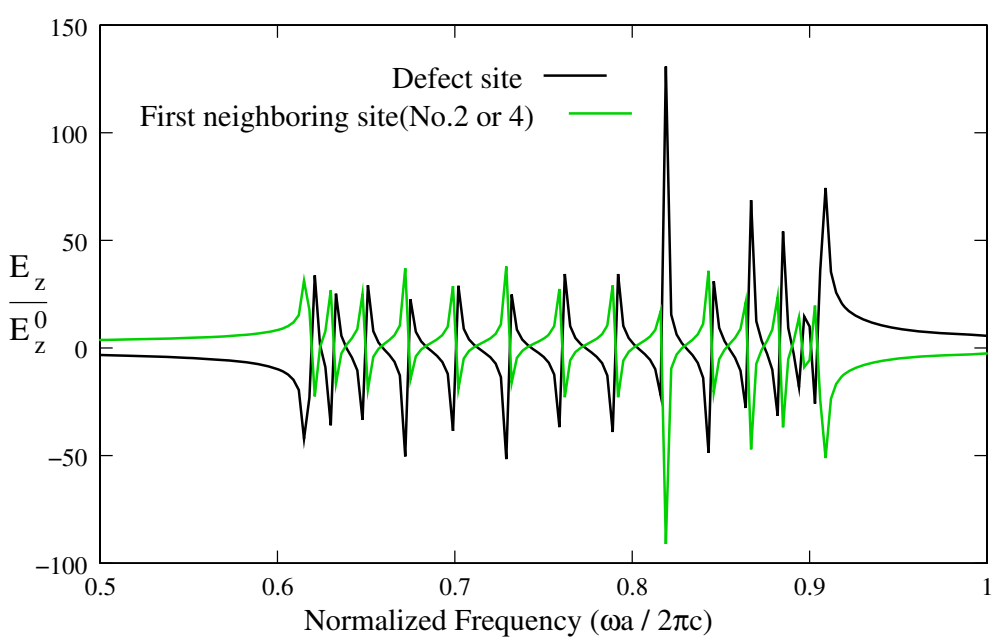

Figure $10 \mathrm{~A}$ comparison of electric fields for different sites. The ratio of electric field of defect site (black line) and the first neighboring site on upside or downside of the defect (green line) are compared which indicates different signs of electric field for all range of frequency. $k_{x}$ is constant corresponding to the first BZ edge.

\section{Application of the method to a PC slab}

In order to relate the wave function of a defected system to a perfect system, Equation 26 is rewritten while a perturbation term is included:

$$
\widehat{\psi}_{l}^{\alpha}={\widehat{\psi^{0}}}_{l}^{\alpha}+\sum_{l^{\prime}, l^{\prime \prime} ; \beta, \gamma} \widehat{G}_{l, l^{\prime}}^{\alpha, \beta}\left(\varepsilon_{l^{\prime}}^{\beta} \delta_{l^{\prime}, l^{\prime}}^{\beta, \gamma}-\delta t_{l^{\prime}, l^{\prime \prime}}^{\beta, \gamma}\right){\widehat{\psi^{0}}}_{l^{\prime \prime}}^{\gamma}
$$

We apply this model for a quasi-one-dimensional PC slab consisting of periodic dielectric rods in which the dielectric constant $\varepsilon_{r}=8.9$ and the central defect site is an air cylinder with a circle cross section. The slab is infinite along the $x$ direction, but due to layering highdielectric-constant material sheets on its borders parallel to the $x$ direction, it has a finite $y$ direction. Although we have considered the first neighboring sites, the difference of the dielectric constant term for neighboring sites, $\delta t_{l^{\prime}, l^{\prime \prime}}^{\beta,}$, can be generalized in such a way that other neighboring sites can be included. For numerical calculation of $\widehat{G}_{l, l^{\prime}}^{\alpha, \beta}$, Equation 20 or 22 is used in which due to the action of the photon creation and annihilation operators $a_{l}^{\alpha \dagger}$ and $a_{l}^{\alpha}$ on the center of sites, $d_{l}^{\alpha}$ is the distance between the center of the sites. The quantized parameter $v$ for $k_{y}=\frac{\pi v}{w}$ is summed over all discrete modes of separate sites on the $y$ direction. Figure $3 \mathrm{a}$,b shows plots of dispersion relation in terms of $k_{x}$ presented for two different numerical integers $v=0,1,2,3,4$ and $v=1,2,3,4,5$, respectively. Mode

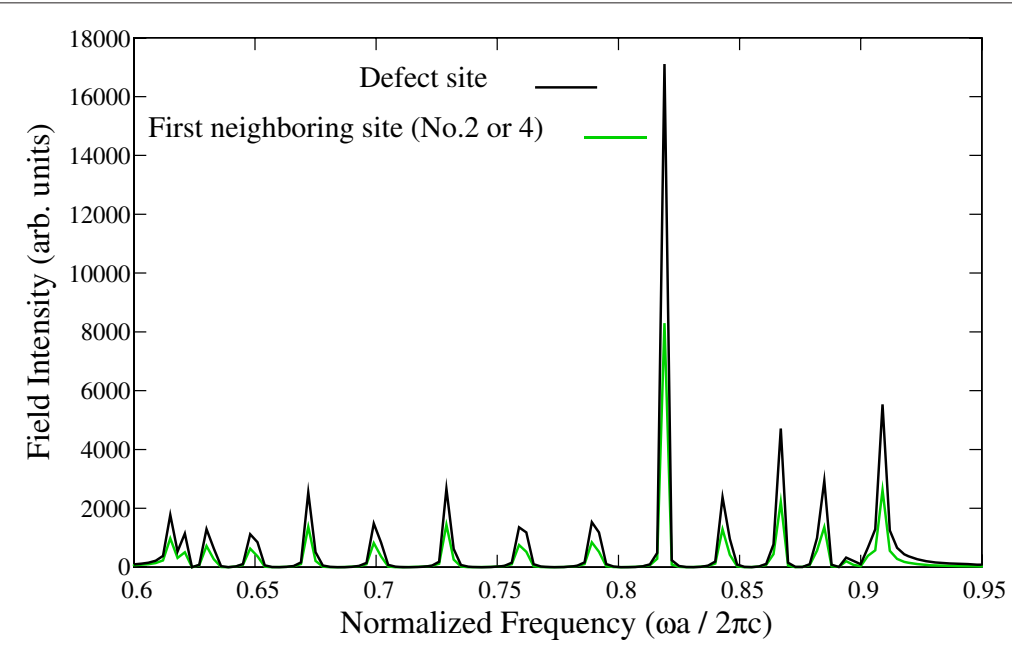

Figure 11 A comparison of the ratio of field intensity for different sites. The ratio of electric field intensity of defect site (black line) and the first neighboring site on upside or downside of the defect (green line) are compared which indicates that the maximum field intensity of the neighboring site and defect site are at the same frequency. $k_{x}$ is constant corresponding to the first BZ edge. 


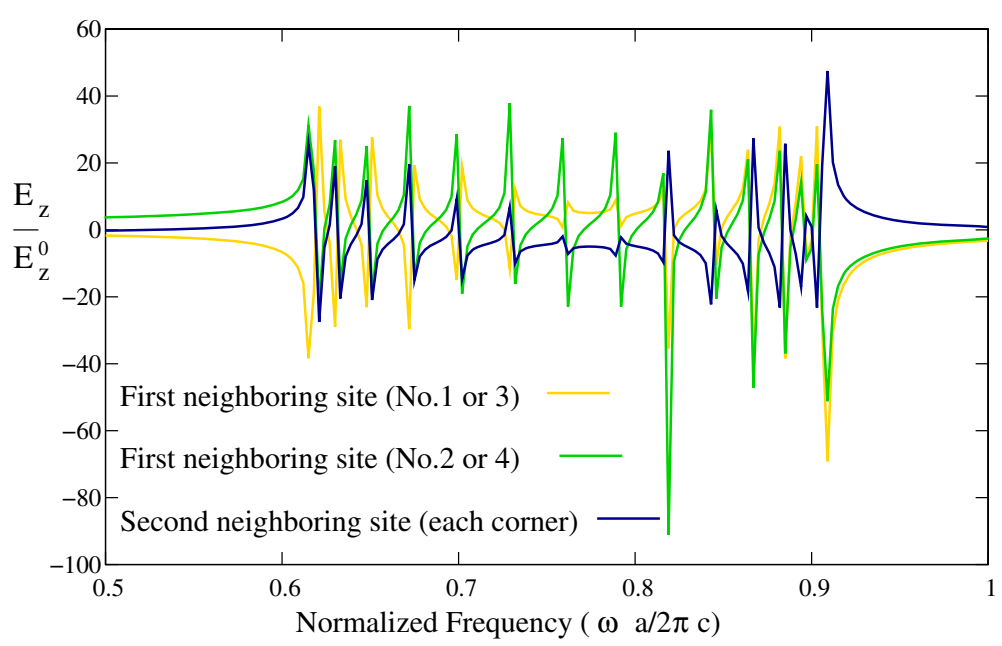

Figure 12 A comparison of electric fields for different sites. The similarities and differences of the sign and amount of the electric field at three sites are compared: the first neighboring site of the right or left (yellow line), the first neighboring site on upside or downside of the defect (green line), and the second neighboring site on each corner side of the defect (dark blue line). $k_{x}$ is constant corresponding to the first BZ edge.

numbers start upward to higher values. There is no degeneracy for modes in Figure 3a, but since the third and fifth modes are degenerate, in Figure $3 \mathrm{~b}$ four modes are plotted. We calculated the electric field of the defected system at different sub-sites for a slab with five sub-sites. Figure 4 shows the ratio of electric field, and Figure 5 shows the ratio of electric field intensity with respect to its clean system at defect site $l=0$ in sub-site $\alpha=3$ (central site). In Figure 4 at normalized frequency 0.819 , the peak's height is maximum 10.2, while it is 103 for the ratio of field intensity which is presented in Figure 5, where these peaks and other peaks in the following figures correspond to the first Brillouin zone (BZ) edges. The ratio of field intensity for site $l= \pm 1$ and sub-site $\alpha=3$ (right side or left side of the defect site) is presented in Figure 6. The peak's height is maximum 10 at normalized frequency 0.909 . The following figures are presented for the second case with $v=1,2,3,4,5$. Figure 7 shows the ratio of field intensity for the defect site; the peak's height is maximum 4,782 where the ratio of $\mathrm{z}$-component of electric field is -69.2 at normalized frequency 0.909 . These are a considerable amount for amplification of incoming pulses at a certain frequency and demonstrate bosonic localization because of the defect and the limited boundaries. The defect disturbs the electric field of other sites which depends on frequency, so a comparison between

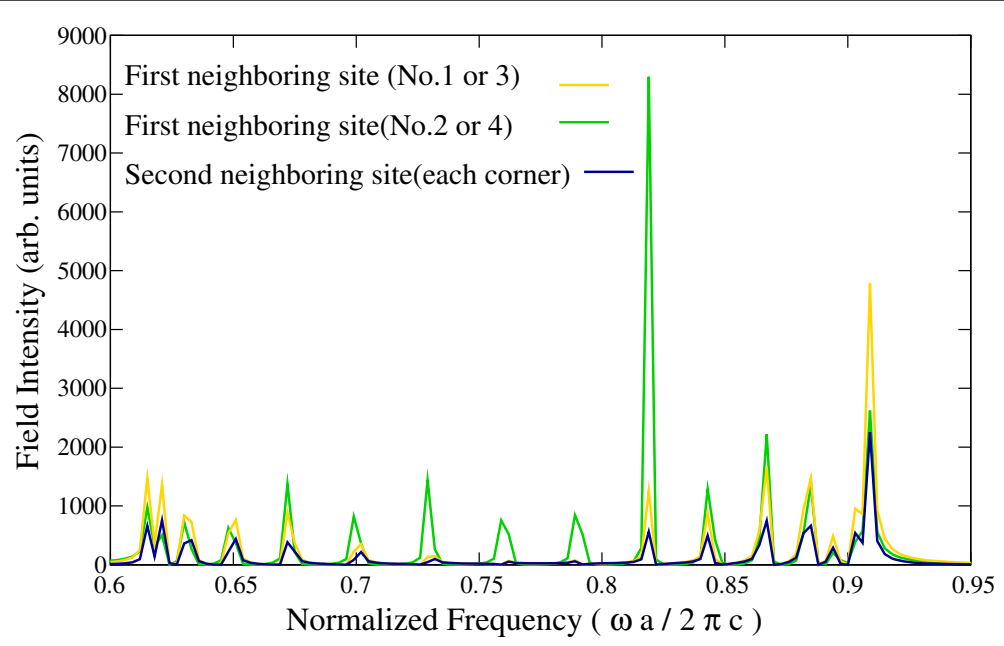

Figure 13 A comparison of the ratio of field intensity for different sites. The ratio of electric field intensity of the first neighboring site of the right or left (yellow line), the first neighboring site on upside or downside of the defect (green line), and the second neighboring site on each corner side of the defect (dark blue line) is compared. $k_{x}$ is constant corresponding to the first BZ edge. 
the ratio of electric field and field intensity of the defect site with the neighboring sites is shown in the following figures where $k_{x}$ takes a constant value corresponding to the first BZ edge. This comparison in Figures 8 and 9 are for the neighboring sites that are indicated by numbers 1 and 3 in Figure $1 \mathrm{~b}$ which are laid on the $x$ direction having the same situation for the ratio of electric field and field intensity. According to Figure 8, an important result is achieved, showing equality of electric field sign of the sites with defect sites for lower frequencies but difference with opposite sign for higher frequencies. Figures 10 and 11 show this comparison for the neighboring sites that are indicated by numbers 2 and 4 in Figure $1 \mathrm{~b}$ and are laid in the $y$ direction. But different from the first neighboring site on the $x$ direction, Figure 10 shows that the electrical field of these sites have opposite sign with the electric field of the defect site for both the lower and higher frequency range. In Figures 12 and 13, electrical field and field intensity of the second neighboring site on each corner of the defect site are compared with those of the two mentioned first neighboring sites. As it is evident in Figure 12, these sites have different electrical field with opposite sign with the first neighboring site in the $x$ direction (no. 1 or 3 sites) but with equality sign for lower frequencies and opposite sign for higher frequencies with the first neighboring site in the $y$ direction (no. 2 or 4 sites).

\section{Results and discussion}

Based on the first-order perturbation theory and Lippmann-Schwinger formalism, we have presented a new treatment for light transmission through a PC slab which introduces a new equation by including disorders of the system. The equation has a general form and is applicable to 2D PC systems with different polarizations, but we simplify it for TM modes and extend the method to investigate effects of a point defect on the light transmission along a boundary-limited slab length in which the slab system is mapped to a quasi-1D system. The vanishing electric field at the boundaries due to total reflection leads to quantization of a photon wave vector component perpendicular to the incident direction. By discretizing Maxwell's equations and mapping them to a tight binding model for the perfect system, the proper dispersion relation and the slab Green function are obtained. This is a theoretical approach similar to electronic semiconductor calculation; by then we have introduced a scheme which realizes localization of photons and precise calculations site by site in the slab. For this purpose, we have investigated the effects of a central defect on light propagation, and we find out that the electric field at the defect site is maximized for the first BZ edges. Therefore, realization of photon localization in the quasi-1D defected PC slab is maintained. The ratio of the electrical field at the first neighboring site parallel to incoming modes is compared with the defect site which indicates that the electric field sign for lower frequencies is the same but is different for higher frequencies with maximum field intensity at higher frequencies with respect to the defect site. On the other hand, the ratio for the first neighboring sites with a perpendicular direction to incoming modes indicates a different sign of electric field for all ranges of frequencies but maximum field intensity at the same frequency with respect to the defect site. Finally, the same procedure is plotted for the second neighboring site on the corners of the defect site and is compared with the previously mentioned first neighboring site.

\section{Conclusions}

Using Lippmann-Schwinger formalism and tight binding approach, we calculate the field intensity in a photonic crystal structure. The method of tight binding can be an appropriate way to calculate the localization of light as well as an efficient way in investigating disorders in the photonic crystals. We have introduced a new equation by including disorders of the system in a general form. In a designed system with a defect in the central site while the boundaries are limited, we have demonstrated that the enhancement of the electric field intensity takes place in the cavity-like defect site which can be applicable in frequency filtering or resonating cavity studies. The calculation is also done for the first and second neighboring sites of the defect site, and the results are compared with the defect site in which considerable differences are obtained.

Competing interests

The authors declare that they have no competing interests.

\section{Authors' contributions}

RM and JS contributed equally in this article. Both authors read and approved the final manuscript.

\section{Acknowledgements}

The corresponding author gratefully acknowledges Soroush Rostamigooran for his language editing.

\section{Author details}

1 Department of Physics, Faculty of Science, Razi University, Kermanshah 67149-67346, Iran. ${ }^{2}$ Nano Science and Technology Research Center, Razi University, Kermanshah 67149-67346, Iran. ${ }^{3}$ Computational Physical Science Research Laboratory, Department of Nano-Science, Institute for Studies in Theoretical Physics and Mathematics (IPM), P.O. Box 19395-5531, Tehran, Iran.

Received: 31 October 2012 Accepted: 4 April 2013 Published: 24 April 2013

\section{References}

1. Yablonovitch, E: Inhibited spontaneous emission in solid-state physics and electronics. Phys. Rev. Lett. 58, 2059 (1987)

2. John, S: Strong localization of photons in certain disordered dielectric superlattices. Phys. Rev. Lett. 58, 2486 (1987)

3. Vignolini, S, Riboli, F, Intonti, F, Belotti, M, Gurioli, M, Chen, Y, Colocci, M, Andreani, L, Wiersma, D: Local nanofluidic light sources in silicon photonic crystal microcavities. Phys. Rev. E. 78, 045603(R) (2008) 
4. Topolancik, J, llic, B, Vollmer, F: Experimental observation of strong photon localization in disordered photonic crystal waveguides. Phys. Rev. Lett. 99, 253901 (2007)

5. $\mathrm{Xu}, \mathrm{T}, \mathrm{Zhu}, \mathrm{N}, \mathrm{Xu}, \mathrm{M}$, Wosinski, L, Aitchison, J, Ruda, H: A pillar-array based two-dimensional photonic crystal microcavity. Appl. Phys. Lett. 94, 241110 (2009)

6. Galli, M, Portalupi, S, Belotti, M, Andreani, L, O'Faolain, L, Krauss, T: Light scattering and Fano resonances in high-Q photonic crystal nanocavities Appl. Phys. Lett. 94, 071101 (2009)

7. Kong, X, Liu, S, Zhang, H, Guan, H: The effect of random variations of structure parameters on photonic band gaps of one-dimensional plasma photonic crystals. Optics Communication. 2915, 284 (2011)

8. Hong, L, Chen, H, Qiu, X: Band-gap extension of disordered 1D binary photonic crystals. Physica. B. 297, 164 (2000)

9. Xu, X: Photon localization in amorphous photonic crystal. Appl. Phys. B. 86,467 (2007)

10. Dossou, K, Botten, L, McPhedran, R, Poulton, C, Asatryan, A, Martijn de Sterke, C: Shallow defect states in two-dimensional photonic crystals. Phys. Rev. A. 77, 063839 (2009)

11. Kristensen, P, Mork, J, Lodahl, P, Hughes, S: Decay dynamics of radiatively coupled quantum dots in photonic crystal slabs. Phys. Rev. B. 83, 075305 (2011)

12. Rahachou, A, Zozoulenko, I: Light propagation in finite and infinite photonic crystals: the recursive Green's function technique. Phys. Rev. B. 72, 155117 (2005)

13. Fussell, D, Dignam, M: Quasimode-projection approach to quantum-dot-photon interactions in photonic-crystal-slab coupled-cavity systems. Phys. Rev. A. 77, 053805 (2008)

14. Na, N, Utsunomiya, S, Tian, L, Yamamoto, Y: Strongly correlated polaritons in a two-dimensional array of photonic crystal microcavities. Phys. Rev. A. 77, $031803(2008)$

15. Garcia-Martin, A, Hermann, D, Hagmann, F, Busch, K, Wolfle, P: Defect computations in photonic crystals: a solid state theoretical approach. Nanotechnology. 14, 177 (2003)

16. Wubs, M, Suttorp, L, Lagendijk, A: Spontaneous-emission rates in finite photonic crystals of plane scatterers. Phys. Rev. E. 69, 016616 (2004)

17. Xu, G, Moreau, V, Chassagneux, Y, Bousseksou, A, Colombelli, R, Patriarche, G, Beaudoin, G, Sagnes, I: Surface-emitting quantum cascade lasers with metallic photonic-crystal resonators. Appl. Phys. Lett. 94, 221101 (2009)

18. Kim, T, Lee, S, Kim, M, Park, H, Kim, J: Experimental demonstration of reflection minimization at two-dimensional photonic crystal interfaces via antireflection structures. Appl. Phys. Lett. 95, 011119 (2009)

19. Joannopoulos, J, Johnson, S, Winn, J, Meade, R: Photonic Crystals: Molding the Flow of Light. Princeton University Press, Princeton (2008)

20. Skorobogatiy, M, Yang, J: Fundamentals of Photonic Crystal Guiding. Cambridge University Press, New York (2009)

21. Economou, E: Green's Function in Quantum Physics. Springer, New York (1979)

doi:10.1186/2228-5326-3-27

Cite this article as: Moradian and Samadi: Frequency comparison of light transmission in a defected quasi-one-dimensional photonic crystal slab. International Nano Letters 2013 3:27.

\section{Submit your manuscript to a SpringerOpen ${ }^{\circ}$ journal and benefit from:}

- Convenient online submission

- Rigorous peer review

- Immediate publication on acceptance

- Open access: articles freely available online

- High visibility within the field

- Retaining the copyright to your article

Submit your next manuscript at $\boldsymbol{\nabla}$ springeropen.com 